\title{
Chikungunya Virus Infection: An Update on Joint Manifestations and Management
}

\author{
Maria Krutikov, M.B.Ch.B., M.R.C.P. (UK) ${ }^{1 *}$ and Jessica Manson, M.B.Ch.B., \\ Ph.D., F.R.C.P. ${ }^{2}$ \\ 'Department of Infectious Diseases, University College London Hospital, London, UK; and Department of \\ Rheumatology, University College London Hospital, London, UK
}

\begin{abstract}
ABST RACT
The adv ent of sophisticated diagnostics has enabled the discovery of previously unknown arthropod-borne viruses like Chikungunya. This infection has become increasingly prevalent in the last 10 years across the Indian Ocean and has been brought to media attention by a recent outbreak in the Caribbean. Theoutbreak has been aided by a drastic rise in air travel, allowing infected individuals to transport the virus to pre viously unaffected regions. In addition, a recently documented viral mutation has allowed its transmission by the Aedes albopictus mosquito, therefore facilitating outbreaks in Southern Europe and the USA. The duration and extent of the arthritis seen peri- and postinfection has become a topic of academic interest. Although published data are largely observational, there has been a definite increase in original research focusing on this. Sy mptoms can persist for years, particularly in older patients with p re-existing medical conditions. The etiology is still not fully understood, but viral persistence and immune activation within synovialfluid have been shown in mouse models. There have been no prospective clinical trials of treatment in humans; however, animaltrials are in process. The mainstay of treatment remains anti-inflammatories and steroids where necessary. The clinical presentation seems to mimic common rheumatological conditions like rheumatoid arthritis; therefore recent recommendations suggest the use disease-modifying agents as a common practice for the specific syndrome. This review uses recent published data and draws on our own clinical ex perience to provide an overview of joint complications of Chikunguny a infection.
\end{abstract}

KEY WORDS: Arthritis, arthropod-bornevirus, Chikungunya

\footnotetext{
Abbreviations: CHIKV, Ckikungunya virus; CIR, chronic inflammatory rheumatism.

Cit ation: Krutikov M, Manson J. Chikungunya Virus In fection: An Update on Joint Ma nifestations and Ma nagem ent. Ram bam Maimonides Med J 2 016;7 (4):eoo33. doi:1 0.5041/RMMJ.1026o Review

Copy right: (C) 2016 Krutikov and Ma nson. This is an open-access article. All its content, except where otherwise noted, is distributed under the terms of the Creative Com mons Attribution License (http://creativecom mons.org/licenses/by/3.0), wh ich permits unrestricted u se, distribution, and reproduction in any medium, provided the original work is properly cited.
}

Conflict of interest: No potential conflict of in terest relevant to this article was reported.

* Tow hom correspondence sh ould be a ddressed. E-mail: maria.krutikov @nhs.net 


\section{INT RODUCTION}

Chikunguny a virus (CHIKV) is a mosquito-borne alpha virus that has precipitated several large outbreaks across the southern hemisphere in the last decade. First described in Tanzania in the 1950s, Chikungunya, also known as break-bone fever, means "that which bends up" in the Makonde language. Following a few outbreaks in the $1960 \mathrm{~s}$ and 1970 s in Asia and Africa, the virus re-emerged in 2005 and spread across the Indian Ocean. Since 2014, over a million cases have been reported in the Americas and the Caribbean, with declining case numbers in 2016. Much of what we currently know about the disease was first published following a large outbreak on the island of La Réunion, affecting $37 \%$ of the entire population. ${ }^{1}$

The virus causes a self-limiting acute illness comprising of fever, rash, arthralgia, and my algia. Other complications, like multi-organ failure and neurological manifestations, have been described but are ex tremely rare. The most notable long-term complication is a chronic debilitating arthritis that causes considerable disability in certain groups. As we gain more experience with this disease, published literature describing this manifestation has become more plentiful; however, little is understood about the pathophysiology and optimal management of this condition. This review will provide an update of current data and recommendations on Chikungunya-related arthralgia.

\section{THEVECTOR}

Chikunguny a is a zoonotic arbovirus that is transmitted by the Aedes mosquito. These are day-biting arthropods that breed in open water and can be found across Africa and Asia. Epidemics occur through human-mosquito transmission made possible by high-circulating viremia during acute infection. Between epidemics, the life cycle is maintained through primates and mainly Aedes aegypti mosquito. The genetic variation seen in this RNA virus has allowed mutation of the virus to enable transmission by the Aedes albopictus, an arthropod that is well established in the Americas andEurope. ${ }^{2}$ This has facilitated the outbreaks of the last 10 years, around the Indian Ocean and across the Caribbean, with an outbreak in Italy in 2007 and cases in other parts of Europe. 3 Subsequently, there are concerns that the virus may, in time, cause further outbreaks across Europe and Northern America.

\section{DIAGNOST ICS}

Diagnostic techniques have improved significantly in recent years and can be grouped into molecular and serological tests.

Molecular tests are most useful in the acute phase of illness. These use reverse transcriptase PCR assays to amplify fragments of the CHI KV genome and can be used to quantify fragments in real time. $4 \mathrm{~A}$ development in recenty ears has been the multiplex assay on a chip that can simultaneously detect 26 tropical pathogens including $\mathrm{CHIKV}$ and dengue. 5

Serological assays can be used to determine whether the patient has been previously infected. These use enzyme-linked immunosorbent assays (ELISAs) that capture IgM and IgG. Other tests include immunofluorescence and immunoblot assays for CHIKV proteins. 4 More recently a rapid immunochromatographic test has been developed that can detect CHI KV , best used in the first 5 days of illness, with a sensitivity of $89.4 \%$ and specificity of $94.4 \%{ }^{6}$ This would allow rapid diagnosis at the point of care, therefore precluding the need for further investigations.

\section{CLINI CAL COURSE OF T HE DISEASE}

The clinical illness has a relatively short incubation period-ty pically 2-10 days. Sudden-onset high fever is usually the first symptom and can last up to a week and can follow a biphasic course. Feveroften precedes a maculo-papular rash over the trunk and extremities, headache, myalgia, and arthralgia. Ocular disease has been described in Malaysia from a few cases of anterior uveitis. 7 More severe complications include nephritis, myocarditis, meningo encephalitis, Guillain-Barré syndrome, and cranial nerve palsies that can occur during the acuteillness or in subsequent months. 4 It can be difficult to differentiate acute CHIKV infection from dengue; however, it has been suggested that thrombocy to penia is more indicative of dengue, whereas rash and arthralgia suggest CHIKV. ${ }^{8}$ It has also been noted that pregnant women infected with the virus in the last few day s of pregnancy are able totransmit the virus to the baby, which can lead to severe encephalopathy in the neonate and subsequentneurodisability, as demonstrated in a recent cohortstudy from La Réunion. 9

After the acute illness, which can last from 7 to 10 days, rheumatic sequelae can persist for months 
to y ears. These can have broad clinical presentations and an, as yet, unclear etiology.

\section{CHI KUNGUNYA RHEUMAT ISM}

Musculoskeletal manifestations of diseasehavebeen shown to affect $4 \%-75 \%$ of those infected with CHIKV.4 These figures vary widely depending on baseline genetic susceptibility of populations, cultural perceptions, and quality of study.

Arthralgia usually affects more than one joint, particularly knees, ankles, hands, and wrists in a bilateral and symmetric distribution. ${ }^{10}$ In some cohorts, over $50 \%$ of patients had arthralgia and clinically detectablejoint swelling at 3 years after their acuteinfection. ${ }^{11,12}$ A 6-year retrospectivestudy in La Réunion by Javelle et al. looked at patients referred to a rheumatologist due to rheumatic symptoms lasting more than 4 months following CHI KV infection. Out of 159 cases, they found that $59 \%$ met the criteria for de novo chronic inflammatory rheumatism (CIR) like rheumatoid arthritis, spondy larthropathy, and undifferentiated polyarthritis, and $31 \%$ had pre-existing rheumatic musculoskeletal disorders. Amongst those with de novo rheumatoid arthritis, $80 \%$ developed joint damage within 3-4 years. They found that some patients remained sy mptomatic for 6-8 years. Patients that did not fit the CIR criteria presented most commonly with bilateral distal poly arthralgia, fibromy algia, edema, and carpaltunnel syndrome.13This finding was mirrored in a study from Sri Lanka from 2006 where $21 \%$ of the total number of infected individuals exhibited carpal tunnel syndrome.14 There have also been recent cases of patients presenting with catastrophic antiphospholipid syndrome and adult-onset Still's disease. ${ }^{15}$

Plasma markers of inflammation such as ESR and CRP are unreliable in predicting the severity of joint involvement. Javelle et al. found thatonly 2 out of 15 patients with spondylarthropathies were HLAB27 positive, both of whom had psoriasis. Rheumatoid factor (RF) or anti-citrullinated protein antibody (ACPA) positivity was found in 30\% of patients, which was similar to other studies. ${ }^{13}$

Radiological imaging of affected joints showed erosions and joint space narrowing in a smallcohort in La Réunion. ${ }^{16}$ Destructive lesions were more commonly seen in those with an rheumatoid arthritislike presentation, with up to $80 \%$ affected in some cohorts. ${ }^{13}$ By contrast, in our London cohort of $\mathrm{CHIKV}$-infected returning travelers, we found pre- dominantly joint effusions rather than synovitis on ultrasound examination (unpublished data). Magnetic resonance imaging has similar findings to ultrasound with predominant joint effusion, synovial thickening, tendonitis, and tenosy novitis. ${ }^{17}$

Risk factors for prolonged disease are agegreater than 50 years, longer acute stage of illness (more than 15 days), and presence of other comorbidities. ${ }^{18}$ A study looking at predictors of rheumatism in the TELECHIK cohort of patients in La Réunion performed a multinomial logistic regression on the 346 patients with declared rheumatic musculoskeletal pain. They found age greater than 45 , severe rheumatic involvement at presentation (fever, more than six joints affected, and four other rheumatic symptoms), and CHIKV-specific IgG titers were strong determinants of persistent musculoskeletal pain. The CHI KV-specific IgG titers were correlated to age, female gender, and severity of initial rheumatic sy mptoms. They concluded that the humoral immune response has a significant prognosticvalue; however, the adaptive immune response plays an important role in chronic manifestations. ${ }^{10}$

The etiology of the arthralgia is not fully understood. Research from mouse models comparing wild-type and Rag1-deficient mice that lack T and B cells inoculated with CHIKV shows higher viral levels in Rag1-deficient mice. This suggests the role of the adaptive immune system in host response and persistence of infection. In addition, tissues with high CHI KV RNA lev els in these mice histologically exhibited synovitis, arthritis, and tendonitis, suggesting that the arthritis is not mediated by the adaptive immune system. The persistence of CHIKV was tissue-specific as sampling of other organs showed rapid clearance of the virus when compared with joints and skeletal muscle, in which it persisted for at least 16 weeks. ${ }^{19}$

Samples from a human synovial biopsy taken from an affected individual with chronic joint disease showed joint infiltration with natural killer cells, CD4 cells, and CHIKV RNA in macrophages. Histologically there was sy novial hypertrophy, vascular proliferation, and perivascular macrophage infiltration similar to that seen in rheumatoid arthritis. ${ }^{20}$ This suggests that the CHI KV arthralgia is etiologically similar to other inflammatory arthropathies and, as such, a similar treatment approach can be adopted.

As disease can last for years, there is evidence that quality oflife of individuals is adversely affect- 
ed. A study by Couturier et al. followed up CHI KV infected individuals for 2 y ears following diagnosis. Of the 391 patients that answered thesurvey, $55 \%$ of patients considered themselves to have not recovered from $\mathrm{CHIKV}$ at a median of 23.4 months post diagnosis. Assessments of quality of life using well-known tools like SF-36 (short term health survey), GHQ-12 (general health questionnaire), and AIMS2-SF (arthritis impact measurement scale) were performed at regular intervals and compared with age- and gender-matched scores. Those who considered themselves as unrecovered had much lower scores than recovered participants. ${ }^{18}$ In addition, there is evidence of the financial burden that this can have-a study from La Réunion has estimated the annual cost at $€_{34}$ million. ${ }^{12}$

\section{T REATMENT AND PREVENTION OF INFECTION}

There are currently no licensed treatments for CHIKV infection. Several drugs have been found to have modest effect by targeting viral replication or host cellular components. Although these mainly target acute viral infection, little is known of the effect of antiviral treatments on chronic infection.

Drugs that inhibit viral entry include chloroquine and arbidol. Chloroquine, historically an antimalarial drug, has been thought to be effective against chronic CHIKV since the early 1980s; however, a recent trial using it as treatment in acute infection has shown it to be ineffective. ${ }^{21,22}$ Arbidol is a licensed antiviral in Russia, and arbidol analogues have been found to have anti-CHIKV activity. 23

A potent inhibitor of viral protein translation has been found to be harringtonine and its more stable analogue, homoharringtonine. This drug has minimal cy totoxic effects and has recently been licensed in the USA for treatment of acute myeloid leukemia. ${ }^{24}$

Most effective of the antiviral drugs are those that target viral genome replication. Ribavirin has been used most widely as it is licensed for treatment of respiratory syncytialvirus in infants. Ribavirin in combination with interferon has been shown to act sy nergistically in preventing CHI KV replication. 25

Mycophenolic acid appears to be more effective than ribavirin in vitro as described by Khan et al. in 2011. ${ }^{26}$ A nother anti-metabolite that is effective in vitro is 6-azauridine as it inhibits synthesis of pyrimidines. ${ }^{22,25}$ Favipiravir has been shown to protect mice against $\mathrm{CHIKV}$-caused disease as a potent polymerase inhibitor. ${ }^{27}$

Although these drugs have displayed some efficacy, they can only reduce viral replication by $50 \%$ in mouse models and have not yet moved to morerelevant testing platforms for chronic CHI KV infection.

An alternative to these antiviral drugs is human poly-or monoclonal antibodies. The use of human polyvalent antibodies taken from human plasma donors in the convalescent phase of CHIKV infection showed full protective efficacy in mouse models. This effect was completely therapeutic 8 hours post infection and reduced as time passed. ${ }^{27}$ Prophylactic administration of neutralizing monoclonal antibodies to Rag1-deficient mice prevented them from developing persistent infection and was even effective in clearing CHI KV from tissues when administered later on in infection. ${ }^{19}$ This work suggests the potential for monoclonal neutralizing antibodies in prevention of CHI KV-associated arthralgia in endemic areas.

Further preventive measures such as vaccines against CHIKV have noty et been licensed but have been a goal since the 1960 . The CHIK-IRES vaccine is an attenuated vaccine derived from a strain of virus from La Réunion in 2006. This has been engineered to prevent it from replicating in the Aedes mosquito and, following recent trials in non-human primates, is planned for phase I trials. Another promising vaccine has implemented induced neutralizing antibodies and virus-like particles (VLPs) which are immunogenic in non-human primates and has entered into phase II trials (NCTo 2562482). ${ }^{27}$ It will be important to show that these vaccines can have long-lasting protection against the virus and are robust against wild-type virus and different vectors.

The release of a genetically engineered mosquito carrying lethal genes (Release of Insects with Dominant Lethal Gene [RIDL]) has also been investigated for prevention of infection. However, there have been concerns that this measure in Aedes aegypti in Panama has allowed the mutation of CHI KV to be transmitted by the Aedes albopictus. ${ }^{28}$ In addition, it may even drive selection of more virulent and severe viruses. ${ }^{2}$ A nother approach has been to infect mosquitoes with the Wolbachia bacterium, which is thought to interfere with mosquito replication. 


\section{MANAGEMENT OF CHIKV RHEUMAT ISM}

Management of CHIKV rheumatic disease has traditionally used a generic approach to this diverse group of clinical presentations. Javelle etal. recently suggested grouping patients according to their clinical sy ndrome and tailoring treatment accordingly. 13

The mainstay of treatment has been with antiinflammatory drugs, physiotherapy, and short courses of oral steroids; however, clinical withdrawal of these treatments can be difficult. Evidence from La Réunion suggested that loco-regional disease responded well to physiotherapy and to localized joint injections. Carpaltunnel syndromewas treated with NSAIDs and with physiotherapy. Uratelowering drugs can be effective, as can bonestrengthening measures such as vitamin D. ${ }^{13}$

In those with persistent symptoms, there is little evidence on effective therapies. Several diseasemodifying drugs (DMARDs) have been trialed with varying success. Chloroquine has some antiviral effect but has not been found to be more effective than other anti-inflammatories like meloxicam in acute and chronic $\mathrm{CHIKV}$ arthralgia. ${ }^{29}$ Methotrexate has been widely used, particularly in patients who present with a systemic polyarthritis. A recentstudy showed that $75 \%$ of patients had a positive clinical response to this. ${ }^{13}$ Sulfasalazine has been shown to have good clinical efficacy, particularly when combined with methotrexate. ${ }^{\circ}$

There are no good-quality trials assessing the use of biologic drugs in CHI KV rheumatic disease. There are data to suggest that serum cytokines are raised in patients with persistent symptoms, including IL-6 and IL-8. ${ }^{31}$ In addition, a number of cases report successful use of biologic immunomodulatory agents such as infliximab or etanercept in patients with severe disease. $3^{2}$

Bindarit is a small-molecule indazolic derivative that has anti-inflammatory properties through inhibiting chemokine synthesis. This has been trialed in CHI KV mouse studies to reduce joint damage and inflammation and improve bonehealth.3334 These studies showed reduced macrophage infiltration of joints and therefore reduced tissue damage and disease symptoms and also reduced osteoclastic activity. As it is less immunosuppressive than more widely used biologic agents such as antiTNF drugs, it has the potential to be a safe treatment to prevent viral-induced joint damage and bone loss.

\section{CONCLUSION}

Since its initial description in the 1950 os, there have been multiple large Chikungunya outbreaks worldwide. Mutation of the virus to allow carriage by Aedes albopictus has enabled transmission of the virus in previously unexposed areas, substantially increasing the at-risk population. The recent warnings in relation to Zika virus have emphasized the increasing risk from Aedes-borne viruses to the general population.

The growing body of evidence regarding the debilitating chronic arthritis following Chikungunya infection has illustrated trends in presentation. It has also allowed certain conclusions to be drawn regarding treatment strategies from the experience of clinicians worldwide. As the arthritis often has a strong semblance to well-described rheumatic presentations, it has often been treated as such, with reasonable outcomes. The importance of maintaining a holistic approach and considering quality of life is evident, particularly as long-term sequelaeare more frequently seen in older patients with preexisting medical comorbidities. Although the mortality associated with this infection is small, the morbidity and burden of disease is large, affecting several million people worldwide. With a rapidly expanding area of endemicity, rheumatologists should be prepared to see more chronic arboviral arthritides in their day-to-day clinics. Moreresearch and awareness is necessary in order to develop better treatment strategies for such patients.

\section{REFERENCES}

1. Roques $\mathrm{P}, \mathrm{Ng}$ LF, Sam IC, Higgs S. Chikungunya: international focus issue. Vector Borne Zoon otic Dis 2015;15:221-2. Full Text

2. Higgs S, V anlandingham D. Chikungunya virus and its m osquitovectors. Vector Borne Zoonotic Dis 2015; 15:231-40. Full Text

3. Rezza G, Nicoletti L, Angelini R, et al. Infection with chikungunya virus in Italy: an outbreak in a tem perate region. Lancet2007;370:1840-6. Full Text

4. Sam IC, Kümmerer BM, Chan YF, Roques P, Drosten C, Abu Bakar S. Updates on chikungunya epidemiology, clinical disease, and diagnostics. Vector Borne Zoon otic Dis 2015;15:223-30. Full Text

5. Tan JJ, Capozzoli M, Sato M, et al. An integrated labon-chip for rapid identification and simultaneous differentiation of tropical pathogens. PLaS Negl Trop Dis 2014;8:e3043. 
6. Lee VJ, Chow A, Zheng X, et al. Simpleclinical and labor a tory predictors of Chikungunya versus dengue infections in adults. PLoS Negl Trop Dis 2012; $6: \mathrm{e} 1786$

7. Gérardin P, Sampériz S, Ramful D, et al. Neurocognitive outcome of children exposed to perinatal mother-to-child Chikungunya viru s infection: the CHIMERE cohort study on Reunion Island. PLoS Negl Trop Dis 2014;8:e2996.

8. Gérardin P, Fianu A, Michault A, et al. Predictor s of Chikunguny a rheumatism: a prognostic survey ancillary to the TELECHIK cohort study. Arthritis Res Ther 2013;15:R9. Full Text

9. Okabayashi T, Sasaki T, Masrinoul P, et al. Detection of chikungunya virus antigen by a novel rapid immunochromatographic test. J Clin Microbiol 2015;53:382-8. Full Text

10. Javelle E, Tiong TH, Leparc-Goffart I, Savini H, Sim on F. Inflammation of the external ear in acute chikungunya infection: experience from the outbreak in Johor Bahru, Malaysia, 2008. J Clin Virol 2014;59:270-3. Full Text

11. Moro ML, Grilli E, Corvetta A, et al. Long-term chikungunya infection clinical manifestations after an outbreakin Italy: a prognostic coh ort study. J Infect 2012;65:165-72. Full Text

12. Schilte C, Staikowsky F, Couderc T, et al. Chikungunyavirus-associated long-term arthralgia: a 36month prospective longitudinal study. PLoS Negl Trop Dis 2013;7:e2137.

13. Javelle E, Ribera A, Degasne I, Gaüzère BA, Marimoutou C, Simon F. Specific management of post-chikungunya rheumatic disorders: a retrospectiv e study of 159 cases in Reu nion Island from 20062012. PLoS Negl Trop Dis 2015;9:eooo3603.

14. Kularatne SA, Weerasinghe SC, Gihan C, et al. Epidemiology, clinical manifestations, and long-term outcom es of a major outbreak of chikunguny a in a ham let in Sri Lanka, in 2007: a longitudinal coh ort study. J Trop Med 2012;201 2:639178. Full Text

15. Betancur JF, Navarro EP, Echeverry A, Moncada PA, Ca-as CA, Tobón GJ. Hyperferritinemia syndrom e: Still's disease and catastrophic antiphospholipid sy ndrome triggered by fulminant Chikungunya infection: a case report of two patients. Clin Rheu matol 2015;34:1989-92. Full Text

16. Bouquillard E, Combe B. A report of 21 cases of rheumatoid arthritis following Chikungunya fever. A mean follow-up of two years. Joint Bone Spine 2009;76:654-7. Full Text

17. Manimunda SP, Vijayachari P, Uppoor R. Clinical progression of chikungunya fever during a cute and chronic arthritic stages and the changes in joint morphology as revealed by imaging. Trans R Soc Trop Med Hy g 2010;104:392-9. Full Text

18. Couturier E, Guillemin F, Mura M, et al. Impaired quality of life after chikungunya virus infection: a 2year follow-up study. Rheumatology (Oxford) $2012 ; 51: 1315^{-22}$. Full Text

19. Hawman DW, Stoermer KA, Montgomery SA, et al. Chronic joint disease cau sed by persistent Chikungunyavirus infection is controlled by the adaptive im m unerespon se. J Virol 2013;87:13878-88. Full $\underline{\text { Text }}$

20. Hoarau JJ, Jaffar Ban djee MC, Krejbich Trotot P, et al. Per sistent chronicinflammation and infection by Chikungunya arthritogenicalphavirus in spite of a robust host immune response. J Im munol 2010 ; 184:5914-27. Full Text

21. De Lamballerie X, Boisson V, Reynier JC, et al. On chikungunya acute infection and chloroquine treatment. Vector Borne Zoonotic Dis 2008;8:837-9. Full Text

22. Abdelnabi R, Neyts J, Delang L. Antiviral strategies against chikungunya virus. Methods Mol Biol 2016;1426:243-53. Full Text

23. Blaising J, Polyak SJ, Pécheur EI. Arbidol as a broadspectrum antiviral: an update. Antiviral Res 2014; 107:84-94. Full Text

24. Kaur P, Thiruchelvan M, Lee RC, et al. Inhibition of chikungunya virus replication by harringtonine, a nov el antiviral that su ppresses viral protein expression. Antimicrob Agents Chemother 2013;57:155-67. Full Text

25. Briolant S, Garin D, Scaramozzino N, Jouan A, Crance JM. In vitroinhibition of Chikungunya and Semliki Forest viruses replication by antiviral com pounds: synergistic effect of interferon-alpha and ribavirin combination. Antiviral Res 2004;61:111-17. Full Text

26. Khan M, Dhanwani R, Patro IK, Rao PV, Parida MM. Cellular IMPDH enzymeactivity is a potential target for the inhibition of Chikungunya virus replication and $v$ irus induced apoptosis in cultured mammalian cells. Antiviral Res 2011;89:1-8. Full Text

27. Ahola T, Couderc T, Ng LF, et al. Therapeutics and $\mathrm{v}$ accines against chikungunya virus. Vector Borne Zoonotic Dis 2015;15:250-7. Full Text

28. Miller MJ, Loaiza JR. Geographic expansion of the inv asive mosquito Aedes albopictus across Panamaim plications for control of dengue and Chikungunya viruses. PLoS Negl Trop Dis 2 015;9:e0oo3383. Full $\underline{\text { Text }}$ 
29. Chopra A, Saluja M, Venugopalan A. Effectiveness of chlor oquine and inflammatory cytokine response in patients with early persistent musculoskeletal pain and arthritis following chikungunya virus infection. Arthritis Rheumatol 2014;66:319-26. Full Text

30. Ganu MA, Ganu AS. Post-chikungunya chronic arthritis-our experience with DMARDs ov er two y ear follow up. J Assoc Physicians India 2 011;59:83-6.

31. Chaaitanya IK, Mu ruganandam N, Sundaram SG, et al. Role of proinflammatory cy tokines and chemokines in chronic arthropathy in CHIKV infection. Viral Immunol 2011;24:265-71. Full Text
32. Blettery M, Brunier L, Polomat K, et al. Management of chronic post-chikungunya rheumatic disease: the Martinican experience.Arthritis Rheumatol 2016 Jun 6. [Epub ahead of print] Full Text

33. Chen W, Foo SS, Taylor A, et al. Bindarit, aninhibitor of monocyte chemotactic protein synthesis, protects against bone loss induced by chikunguny a virus infection. J Virol 2015;89:581-93. Full Text

34. Rulli NE, GuglielmottiA, Mangano G, et al. Amelioration of alphavirus-induced arthritis and myositis in a mou se m odel by treatment with bindarit, an inhibitor of monocyte chemotactic proteins. Arthritis Rheum 2009;60:2513-23. Full Text 